\title{
Linfangioma retroperitoneal neonatal: presentación de dos casos
}

\section{Neonatal retroperitoneal lymphangioma: presentation of two cases}

\author{
Maria Eugenia Cuastumal Cuastumal ${ }^{1,5} \odot$, Jose Gabriel Del Castillo Calderon ${ }^{2} \odot$, Carlos \\ Eduardo Villamil Giraldo ${ }^{3}{ }^{\circ}$, Jose Fernando Gomez Urrego ${ }^{4,5} \mathbb{C}$
}

${ }^{1}$ Universidad Libre Seccional Cali. Facultad de ciencias de la salud. Especialización en pediatría. Cali, Colombia. ${ }^{2}$ Hospital Infantil los Ángeles. Facultad de Ciencias de la Salud. Especialización en pediatría. Pasto, Colombia. ${ }^{3}$ Hospital infantil los Ángeles. Facultad de ciencias de la salud. Especialización en pediatría. Pasto, Colombia.

${ }^{4}$ Universidad Libre Seccional Cali. Facultad de Ciencias de la Salud. Coordinación de especialización en pediatría. Cali, Colombia.

${ }^{5}$ Grupo de investigación en pediatría (GRINPED COL 0142019). Facultad de Ciencias de la Salud. Especialización en pediatría. Cali, Colombia.

\section{RESUMEN}

Los linfangiomas abdominales son tumores quísticos poco frecuentes en pediatría, representan el $1 \%$ de todos los casos de linfangiomas; los síntomas clínicos no son específicos, se descubren de forma incidental; actualmente el manejo quirúrgico es de primera elección, pero se deben valorar otras posibilidades terapéuticas como los agentes esclerosantes que, dependiendo del tamaño, la ausencia o no de sintomatología y las comorbilidades del paciente se convierte en otra opción terapéutica menos invasivo con el cual se logran buenos resultados; se describe los casos clínicos de dos neonatos con diagnóstico de linfangioma retroperitoneal, manejados con agentes esclerosantes (caso 1) y cirugía (caso 2), mostrando las diferentes opciones terapéuticas.

Palabras claves: Linfangioma, doxiciclina, tratamiento, neonatos.

\begin{abstract}
Abdominal lymphangiomas are infrequent cystic tumors in pediatric patients, they represent $1 \%$ of all cases of lymphangiomas; clinical symptoms are not specific, they are usually discovered incidentally; Surgical management is currently the first-line treatment, but other therapeutic possibilities, such as sclerosing agents, must be evaluated. Depending on the size, the presence or not of symptoms and the patient's comorbidities, these alternatives become another less-invasive therapeutic option, with which good results may be achieved. Two clinical cases of neonates diagnosed with retroperitoneal lymphangioma are described, one was managed with sclerosing agents and the other with surgery, exemplifying different therapeutic options.
\end{abstract}

Key words: Lymphangioma, doxycycline, treatment, neonates.

\section{INTRODUCCIÓN}

Los linfangiomas son neoplasias benignas extremadamente raras del sistema linfático ${ }^{(1)}$ consiste en masas de canales linfáticos anormales; ocurren en uno de cada 2000-4000 nacidos vivos ${ }^{(2)}$, los linfangiomas retroperitoneales representan el $1 \%$, este tumor consta de varias cavidades similares a quistes llenas de un líquido seroso, sanguinolento, o quiloso $^{(3)}$.

\footnotetext{
Correspondencia: Maria Eugenia Cuastumal Correo: mar.cuastumal@gmail.com

Recibido:31/12/2020 Aceptado:17/02/2021

Conflicto de interés: Los autores declaran no poseer conflicto de interés.

DOI: https://doi.org/10.31698/ped.48012021012
}

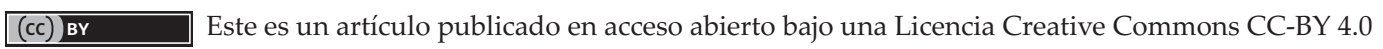


La mayoría suelen ser asintomáticos y se encuentran accidentalmente ${ }^{(1)}$; generalmente se presentan como una masa abdominal palpable, blanda de crecimiento lento e indoloro ${ }^{(4)}$, suelen diagnosticarse en los primeros años de vida y la ecografía fetal puede ser la primera ayuda diagnostica; se confunden fácilmente con otros tumores quísticos retroperitoneales, incluidos los que se originan en el hígado, el riñón y el páncreas, por lo que el diagnóstico representa un desafío; aunque se puede diagnosticar con las imágenes, requiere confirmación histológica ya que el diagnostico diferencial es extenso ${ }^{(5,6)}$.

En cuanto al tratamiento si se usa la escisión debe ser lo más completa posible para reducir el riesgo de recurrencia ${ }^{(7)}$; La laparoscopia podría usarse con éxito para el linfangioma abdominal, incluso en una emergencia; cuando la resección laparoscópica es imposible, se puede discutir la laparotomía o la escleroterapia $^{(8)}$ la cual se considera apropiada para el tratamiento de lesiones quirúrgicamente irresecables ${ }^{(9)}$.

Entre los agentes esclerosantes han mostrado eficacia etanol, tetradecilsulfato de sodio, bleomicina, doxiciclina y ok-432 (picibanil) ${ }^{(10)}$.

\section{PRESENTACION DE CASOS}

\section{CASO 1}

Recién nacido de 37 semanas, producto del primer embarazo, sin evidencia de infecciones perinatales, controles prenatales completos (8), cesárea por hallazgo ecográfico prenatal de displasia renal multiquistica derecha, peso al nacer 3250 gramos talla: $48 \mathrm{~cm}$, Apgar 8/10.

Hospitalizado en unidad neonatal por hallazgos prenatales de displasia multiquistica, examen físico con distensión abdominal y sensación de masa. Ecografía abdominal con lesión expansiva quística multiseptada de contornos lobulados a nivel perirrenal y con expansión al mesogastrio, tejido celular subcutáneo de la cadera y región lumbar derecha, con disminución del parénquima renal, resonancia magnética nuclear abdominopélvica (Figura 1) evidencia malformación primaria congénita benigna del sistema linfático, compatible con linfangioma quístico retroperitoneal iniciándose manejo con solución esclerosante, una ampolla de sulfato de magnesio mas $100 \mathrm{mg}$ de doxiciclina con resolución de la lesión.

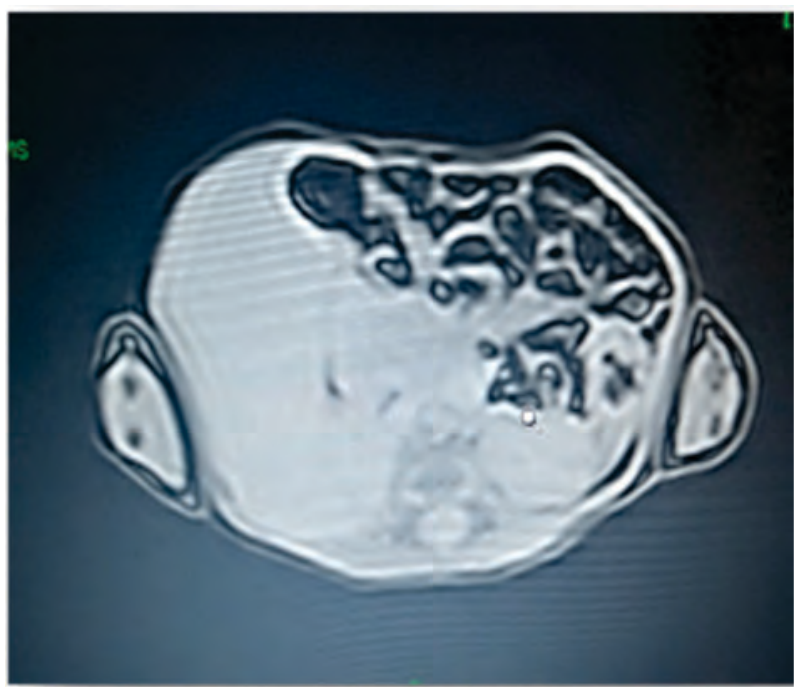

Figura 1. Linfangioma retroperitoneal.

\section{CASO 2}

Recién nacido a término producto de segundo embarazo, sin antecedentes prenatales infecciosos, Apgar 8/10, con ecografía prenatal de hidronefrosis. Se ingresa a cuidado intermedio neonatal por síndrome de dificultad respiratoria, además de distensión abdominal, resonancia magnética (Figura 2) evidencia imagen quística multiseptada que ocupa la cavidad abdomino pélvica y genera efecto compresivo sobre estructuras adyacentes, masa compatible con linfangioma retroperitoneal se lleva a laparotomía exploratoria encontrando gran tumor retroperitoneal izquierdo que desplaza órganos retroperitoneales e intraabdominales, se hace resección de lesión ; paciente es trasladado a cuidado intensivo, recibió ventilación mecánica invasiva por 6 días, en su evolución con sepsis que responde a antibioticoterapia, histología reporta linfangioma retroperitoneal, egresa al día diez. 


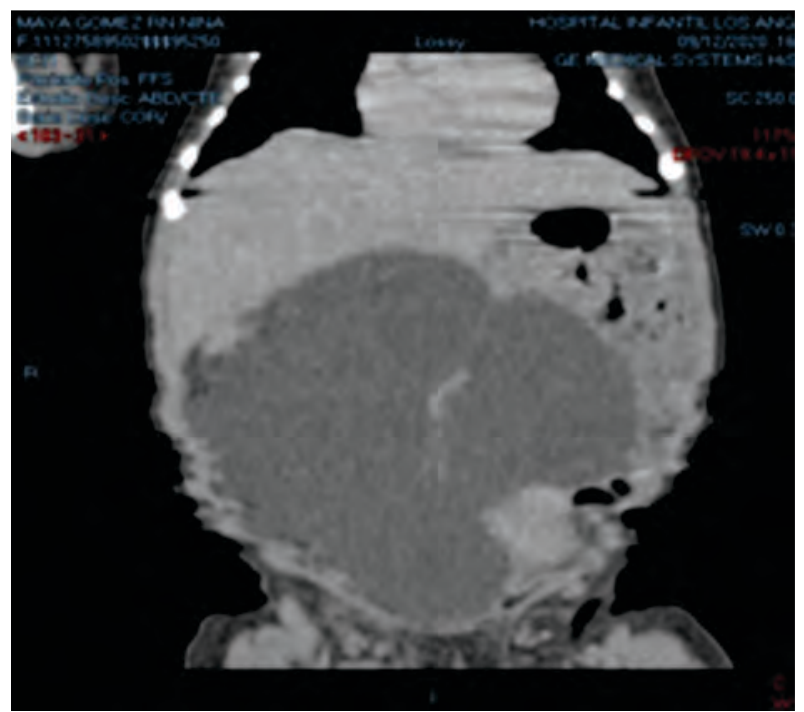

Figura 2. linfagioma retroperitoneal

Tabla 1. Características clínicas y radiológicas

\begin{tabular}{l|l|l}
\hline \multicolumn{1}{c|}{ Característica } & \multicolumn{1}{c}{ Caso 1 } & \multicolumn{1}{c}{ Caso 2 } \\
\hline Edad gestacional (semanas) & 37 & 38 \\
\hline genero & masculino & Femenino \\
\hline Diagnóstico prenatal & Displasia renal multiquistica & Hidronefrosis \\
\hline Topografía de la lesión & Perirrenal derecha & Abdominopélvica \\
\hline Diagnostico imagenológico & $\begin{array}{l}\text { Formación quística compleja } \\
\text { multiseptada retroperitoneal que ingresa } \\
\text { a la gotera parietocolica derecha }\end{array}$ & $\begin{array}{l}\text { imagen multiseptada que mide 96 } \\
\text { x 43mm }\end{array}$ \\
\hline Síntomas y signos postnatales & Masa & $\begin{array}{l}\text { Masa, distensión abdominal, } \\
\text { vómitos persistentes }\end{array}$ \\
\hline Tratamiento & Esclerosis con doxiciclina & Cirugía \\
\hline Seguimiento & No recidiva a 4 meses & No recidiva 3 meses \\
\hline
\end{tabular}

\section{DISCUSIÓN}

Los linfangiomas son anomalías congénitas del sistema linfático, la mayoría se localizan en la cabeza y el cuello, los linfangiomas retroperitoneales representan casi el $1 \%$ de todos los linfangiomas ${ }^{(9)}$. En cuanto a su clasificacion se pueden presentar como macroquisticos, microquisticos y combinados, siendo más frecuente los macroquisticos tal como se reporta en este estudio y el reporte de Gulraiz Chaudry et al. ${ }^{(10)}$.
La mayoría de los casos de linfangioma abdominal son asintomáticos, como lo reporta Muramori et al; ocasionalmente presentan abdomen agudo debido a una obstrucción intestinal o peritonitis causada por quistes infectados, hemorragia o torsión ${ }^{(11)}$. Brian K. et al. reportan la presentación mas aguda en la edad pediátrica en comparación con el adulto ${ }^{(12)}$ tal como se presentó en el caso 2 con síntomas a nivel gastrointestinal. 
En cuanto al diagnóstico no hay un signo especifico y se basa principalmente en la obtención de imágenes con ultrasonido, tomografía computarizada o imágenes de resonancia magnética, posteriormente, debe confirmarse mediante histología ${ }^{(11)}$ tal como se realizó en el caso 2 donde el estudio histológico evidenciola malformación linfática.

Respecto al tratamiento la laparoscopia puede proporcionar un enfoque menos invasivo que laparotomía en la resección de linfangiomas abdominales, cuando la lesión es irresecable o cuando el abordaje percutáneo no es factible, la laparoscopia podría mejorar eficazmente la escleroterapia ayudando a localizar e inyectar las lesiones. sin embargo, si la lesión no se reduce en 3 a 6 semanas después del primer tratamiento, se puede administrar una segunda inyección se reporta un estudio realizado por Muramori et al. ${ }^{(9)}$, Wildhaber et al. ${ }^{(7)}$, en donde se realizó escisión laparoscópica en 2 pacientes de 18 meses y 4 años de linfagiomas quísticos retroperitoneales logrando resección completa sin recurrencias así mismo Bezzola et al., recomienda la escisión quirúrgica total para linfangiomas quístico abdominales sintomáticos ${ }^{(8)}$.

Entre los agentes esclerosantes se han utilizado sustancias esclerosantes como alcohol, bleomicina, ok-43 sin probada eficacia, Chaudry et al. ${ }^{(10)}$ administraron bleomicina en pacientes de 1.5 a 5 años logrando respuesta completa al tratamiento en el $38 \%$ de los pacientes y parcial en el $38 \%$ demostrando su eficacia ${ }^{(10)}$; Amodeo et al. advierten sobre muertes relacionadas con la escleroterapia con bleomicina y fibrosis pulmonar.
En cuanto al ok-432 tiene una tasa de éxito mayor al $80 \%$, causa reacciones sistémicas como fiebre, malestar general, anorexia y no se puede administrar en niños con alergia a la penicilina.

Un estudio de pacientes que se sometieron a escleroterapia con doxiciclina entre 2003 y 2004 en el Children's Hospital Boston en donde se evalúa el cambio en el tamaño de la lesión en 41 pacientes sometidos a escleroterapia, en donde el tipo de lesión más común fue macroquística (49\%), las tasas de complicaciones mayores y menores fueron del $2 \%$ y el $10 \%$, respectivamente, considerando a la doxiciclina como un esclerosante seguro y eficaz ${ }^{(10)}$.

Cahill et al. reportan otras complicaciones de la escleroterapia como anemia hemolítica, acidosis metabólica e hipoglicemia en tres recién nacidos ${ }^{(13)}$.

Amodeo et al. reportan como el manejo conservador en un recién nacido con un linfagioma quístico abdominal macroquisticos permitió la regresión de la masa y evito la intervención quirúrgica ${ }^{(14,5)}$.

Aspectos Éticos: Este trabajo se realizó con autorización del comité de ética del Hospital Infantil los Ángeles de Pasto

\section{AGRADECIMIENTOS}

Agradecemos al Hospital Infantil los Ángeles de Pasto por permitirnos realizar esta investigación. 


\section{REFERENCIAS}

1. Cherk M, Nikfarjam M, Christophi C. Retroperitoneal lymphangioma. Asian J Surg. 2006;29(1):51-4. doi: https://doi.org/10.1016/S1015-9584(09)60297-9

2. Hassan H, Aly KA. Management of cystic lymphangioma: experience of two referral centers. Annals of Pediatric Surgery. 2012;8(4):123-128. doi: https://doi.org/10.1097/ 01.XPS.0000418462.68421.e3

3. Surlin V, Georgescu E, Dumitrescu C, Râmboiu S, Com?nescu MV, Ghilu?i M. Retropancreatic cystic lymphangioma - considerations upon a case. Rom J Morphol Embryol. 2011;52(1Suppl):493-6.

4. Gümüsta OG, Sanal M, Güner O, Tümay V. Retroperitoneal cystic lymphangioma: a diagnostic and surgical challenge. Case Reports in Pediatrics. 2013; 292053. doi: https://doi.org/10.1155/2013/292053

5 Poroes F, Petermann D, Andrejevic-Blant S, Labgaa I, Di Mare L. Pediatric cystic lymphangioma of the retroperitoneum: A case report and review of the literature. Medicine. 2020;99(28):e20827. doi: https://doi.org/ 10.1097/md.0000000000020827

6. Saadi A, Ayed H, Karray O, Kerkeni W, Bouzouita A, Cherif $M$, et al. Le lymphangiome kystique rétropéritonéal: à propos de 5 cas et revue de la littérature. Pan African Medical Journal. 2016;25:73. doi: https://doi.org/10.11604/pamj.2016.25.73.10002

7. Wildhaber BE, Chardot C, Le Coultre C, Genin B. Total laparoscopic excision of retroperitoneal cystic lymphangioma. J Laparoendosc Adv Surg Tech A. 2006;16(5):530-3. doi: https://doi.org/10.1089/lap.2006.16.530

8. Bezzola T, Bühler L, Chardot C, Morel P. Le traitement chirurgical du lymphangiome kystique abdominal chez l'adulte et chez l'enfant. J Chir. 2008;145(3):238-43. doi: https://doi.org/10.1016/s0021-7697(08)73752-9
9. Olivieri C, Nanni L, De Gaetano AM, Manganaro L, Pintus C. Complete Resolution of Retroperitoneal Lymphangioma with a Single Trial of OK-432 in an Infant. Pediatr Neonatol. 2016;57(3):240-3. doi: https:/doi.org/ 10.1016/j.pedneo.2013.06.011

10. Chaudry G, Burrows PE, Padua HM, Dillon BJ, Fishman SJ, Alomari AI. Sclerotherapy of abdominal lymphatic malformations with doxycycline. J Vasc Interv Radiol. 2011;22(10):1431-5. doi: https://doi.org/10.10 16/j.jvir.2011.06.021

11. Muramori K, Zaizen Y, Noguchi S. Abdominal lymphangioma in children: report of three cases. Surg today. 2009; 39:414-417. doi: https://doi.org/10.1007/ s00595-008-3854-z

12. Goh BK, Tan YM, Ong HS, Chui CH. Intra-abdominal and retroperitoneal lymphangiomas in pediatric and adult patients. World J Surg. 2005 ;29(7):837-40. doi: https://doi.org/10.1007/s00268-005-7794-0

13. Cahill AM, Nijs E, Ballah D, Rabinowitz D, Thompson L, Rintoul N, et al. Percutaneous sclerotherapy in neonatal and infant head and neck lymphatic malformations: a single center experience. J Pediatr Surg. 2011;46(11):208395. doi: https://doi.org/10.1016/j.jpedsurg.2011.07.004

14. Amodeo I, Cavallaro G, Raffaeli G, Colombo L, Fumagalli M, Cavalli R, et al. Abdominal cystic lymphangioma in a term newborn: A case report and update of new treatments. Medicine. 2017; 96(8):e5984. doi: https://doi.org/10.1097/MD.0000000000005984 